\title{
LOS LUGARES COTIDIANOS DE LA CULTURA Y EL ARTE
}

Iñaki Domínguez

Universidad del País Vasco

\begin{abstract}
RESUMEN
El objetivo de este trabajo es múltiple: a) Por una parte, proponer una reflexión sobre el espacio que en la vida cotidiana de las sociedades industriales avanzadas está reservado para la relación de los individuos con la cultura y el arte. $b$ ) Como segundo objetivo, pretendemos formular algunas consideraciones sobre el uso público y/o privado de esa relación de los individuos con la cultura y el arte. Para ello, nos proponemos mostrar algunas características de la evolución de ese "espacio temporal» que denominamos «tiempo libre», así como de las variedades y condicionantes del uso que se le otorga en unas sociedades en las que todos los indicios nos señalan el final de un ciclo, el caracterizado en torno al trabajo, entendido tanto como instrumento de desarrollo como mecanismo de diferenciación y significación social. c) Y, finalmente, proponemos un análisis de la inserción de lo cultural en los procesos de diferenciación social, no sólo como resultado de una estratificación social sino, incluso, como una variable independiente que influye significativamente en la construcción y consolidación de la posición social de clase.
\end{abstract}

En las páginas que siguen, nos vamos a referir a la cultura y el arte de una manera genérica, englobando bajo la denominación de cultura a todos los procesos relativos a la creatividad que tienen lugar en las sociedades avanzadas. $\mathrm{Y}$, aunque parezca abusivo no tratar al arte como una «forma» específica de la acción cultural, debe señalarse que el presente artículo se refiere expresamente a la etapa final de todo este proceso, a la relación que los individuos mantienen con las formas artísticas o la creación cultural en su etapa de consumo, cuando

\section{Reis}


la «obra de arte» llega el público y, cada vez en mayor medida, se convierte en un «objeto» a consumir, sea en los museos (cada vez más cercanos a los parques de atracciones y a los lugares de espectáculos), sea a través de los omnipresentes medios de comunicación de masas.

En este sentido, debe consignarse que el arte se acerca y se diluye en la cultura de masas, en un proceso de absorción y uso de lo artístico que el arte y los artistas sólo consiguen evitar o solventar cuando voluntariamente se separan de lo que es fácilmente aprovechable o apropiable por los instrumentos de la cultura de masas, es decir, cuanto más arriesgadas son sus propuestas, cuanto más incierto es su «resultado» o, lo que es casi lo mismo, cuando peligra su consolidación o continuidad como artistas, como actores sociales de la creatividad.

Pero, junto a estas razones que pretenden justificar la utilización del término "cultura" para abordar el problema de la sociología del arte, hay un motivo basado en la propia evolución de la Sociología, en su manera de abordar los procesos culturales y artísticos, sobre todo a partir de la aparición de los «estudios culturales» (cultural studies), con su tendencia a la incorporación de todas las formas creativas como procesos culturales equivalentes. Veámoslo con más detalle.

\section{LA NUEVA ETAPA DE LA SOCIOLOGÍA DE LA CULTURA}

Las sociedades posmodernas mantienen con la cultura una relación compleja en la que se dan cita no sólo la propia complicación de «lo cultural» —un campo caracterizado perennemente por la dificultad de su delimitación y en el que, además, se ha producido una progresiva diversificación de sus componentes, formas y contenidos-, sino también aspectos intrínsecos a su condición posmoderna, como las tendencias de cambio que afectan al papel del trabajo como referente básico en la interpretación de la posición social de los individuos, y la consiguiente aparición de nuevos criterios de estratificación social; o la progresiva alteración de la estructura temporal de la vida cotidiana y el papel que ocupa la cultura en este «nuevo» espacio temporal: el acrecido tiempo libre.

Todo ello ha provocado una multiplicación en las formas de relación que los actores sociales mantienen con la cultura, y obliga a la Sociología a una profunda renovación de sus «modos de ver», de sus conceptualizaciones y planteamientos analíticos para adecuarlos a la reformulación del papel social de la cultura; una nueva posición obtenida gracias, según D. Bell, a la dinamicidady la legitimidad alcanzadas por lo cultural en las sociedades modernas ${ }^{1}$. Este tratamiento al que aludimos constituye una parte de una más amplia modifica-

1 «En primer término, la cultura se ha convertido en el componente más dinámico de nuestra civilización, superando hasta al dinamismo de la tecnología (...) En segundo término, en los últimos 50 años, aproximadamente, se ha producido la legitimación de este impulso cultural» (1987: 45). 
ción de la actitud de la sociedad hacia la cultura: la que soporta el paso hacia un consumo masivo de bienes y servicios culturales, en un proceso en el que, paralelamente a la ampliación del ámbito de difusión del consumo de cultura, se producía una constante y simultánea redefinición de los lujos en necesidades ${ }^{2}$.

En lo relativo a la cultura asistimos, por tanto, a una transformación doble. De un lado, un cambio en la actitud social ante la cultura, que deja de considerarla como un lujo y pasa a aceptarla como un elemento imprescindible para el desarrollo de la vida cotidiana. Pero, también, se ha producido una modificación del papel de la cultura, entendida como sistema significante, esto es, como un sistema diferenciado de otros tipos de organización social (Willians).

Si seguimos a D. Crane (1994), la "sociología de la cultura» ha sido virtualmente reinventada en los años setenta, en un proceso en el que los «estudios culturales» han desempeñado un papel de extremada importancia, no sólo por el reconocimiento de lo cultural como un conjunto de prácticas significantes, sino también por la diversificación de su propio objeto de estudio y, fundamentalmente, por el reconocimiento de la trascendencia de los media en los procesos de interacción social y cultural de las sociedades modernas.

Pese a esta renovación, y como no podía ser de otra manera, esta «nueva etapa» de la sociología de la cultura se articula en torno a la recuperación y replanteamiento de algunas de las cuestiones básicas expresadas en relación a lo cultural:

a) Cómo definir y conceptualizar la cultura en todas sus ramificaciones contemporáneas, tarea tanto más compleja cuanto más cercano se encuentre el concepto de "derechos culturales» a la noción de creación cultural; una proximidad conceptual que fuerza al reconocimiento de simples prácticas de sociabilidad como fórmulas más o menos elaboradas de creatividad, para las que se reclaman identificación, censo y protección en igualdad de condiciones con otras formas de producción y creación cultural ya consolidadas.

b) Cómo conceptualizar las relaciones entre estructura social y cultura en las sociedades de la posmodernidad, es decir, cómo se establecen las relaciones de causalidad entre una y otra esfera, tanto en el continuum idealismo/materialismo como en el grado de autonomía o interdependencia que se "acepta" entre la estructura social y la cultura.

c) Por último, la tercera cuestión que la sociología de la cultura retoma en su formulación más reciente hace referencia a la contingencia de estudiar sistemáticamente a la cultura mediante la aplicación de unos valores sociológicos - los propios de la sociedad del trabajo- incapaces de aprehender y explicar una sociedad en la que el trabajo humano ya no es un referente único de la

${ }^{2} \mathrm{O}$, como dice Bell en ese mismo texto, «la difusión de los que antaño eran considerados lujos a las clases media y baja de la sociedad" (1987: 73), en un proceso que tuvo lugar simultáneamente al de la difusión de los derechos de ciudadanía a esos mismos estratos sociales. 
acción social y en la que las tradicionales nociones de causa-efecto no parecen ser ya relevantes.

La tarea de la sociología de la cultura no es, desde ninguna de estas tres perspectivas de análisis, fácil. La cultura y lo cultural constituyen aspectos centrales del funcionamiento de las sociedades posmodernas, y su complejidad - derivada tanto de la multiplicidad de formas de lo cultural como de la difusión de capacidades tecnológicas que han contribuido a diluir la creatividad y lo cultural en el conjunto de las prácticas de sociabilidad- hace inviable la creación de un modelo único para el análisis, de un modelo de referencia de aplicabilidad universal.

Incluso en el ámbito de las relaciones entre la estructura social y la cultura, las que, según la expresión de D. Bell, se establecen en el mercado, es preciso establecer un conjunto de cautelas en torno a las relaciones de causalidad tradicionalmente consideradas. Así, la propia naturaleza de los vínculos entre "estructura" y "cultura" suscita una respuesta diversa que Rosengren ${ }^{3}$ recoge mediante una doble dicotomía basada en las nociones de determinación e influencia: a) la cultura determina la estructura social (idealismo); $b$ ) la estructura social determina la cultura (materialismo); $c$ ) la cultura y la estructura social no se influyen (autonomía); y d) la cultura y la estructura social se influyen mutuamente (interdependencia).

Obviamente, esta diversidad de respuestas se corresponde con la permanente discusión que las distintas escuelas sociológicas mantienen en torno a la validez de sus respectivos paradigmas. Y, lo que es más importante, se concreta en los análisis desarrollados sobre la estratificación cultural, en sus posibles acepciones: tanto la relativa a la influencia de la estratificación social sobre la relación que los actores sociales sostienen con la cultura - es decir, su capacidad de acceso a la producción, consumo y disfrute de los bienes y servicios culturales - como a la posible incidencia de la cultura como criterio o variable determinante en los procesos de diferenciación social o generación de desigualdades sociales, lo que, a nuestro entender, constituye el núcleo de cualquier análisis sociológico de la cultura que estudie las relaciones entre ésta y la estructura social.

\section{ARTE, CULTURA Y TIEMPO PARA SÍ}

En otra ocasión he tenido la oportunidad de reflexionar sobre los cambios experimentados por la sociedad del trabajo en el transcurso de las últimas décadas $^{4}$. De entre estas transformaciones, cabe destacar el evidente cambio

3 G. MelischeK, K. E. Rosengren y J. STAppers (1984), Cultural indicators: An international symposium", Verlag der Österreichischen Akademie der Wissenschaften, Viena.

${ }_{4}$ «La cultura en los procesos de diferenciación social», comunicación presentada a las 1 .as Jornadas de Sociología y Antropología del Arte: Arte, sociedad, cultura e identidad, organizadas en la Universidad de Buenos Aires (XI-1993). 
habido en la consideración social del trabajo, pero para el objetivo de la presente comunicación quisiéramos poner especial énfasis en otros dos aspectos: uno, íntimamente ligado a la evolución de la relación de los individuos con el trabajo, como es el establecimiento de una nueva estructura temporal de la vida cotidiana; y otro, también relacionado con la evolución del trabajo, aun cuando de una manera más lejana, que resumiremos en torno al concepto de uso del tiempo, es decir, en la distribución socialmente realizada de las actividades que tienen lugar en el espacio temporal que genéricamente conocemos como tiempo libre.

En la actualidad, nos encontramos con un espacio de tiempo que, globalmente entendido, es muy superior al dispuesto por generaciones anteriores y en el que, al margen de la distribución del acceso a ese disfrute de tiempo por las distintas categorías sociales, tema del que nos ocuparemos más adelante, se ha producido la institucionalización de un espacio de tiempo privado, asimilable al espacio físico que el desarrollo de la familia nuclear reconoce y concede a sus integrantes; simultáneo al privilegio de la «intimidad» que la modernidad otorga a sus partícipes.

Es este "tiempo para sí» un espacio impensable en las sociedades premodernas en las que no se haya llevado a cabo un proceso de diferenciación social que permita la individualización y particularización de sus agentes sociales, que admita su derecho a ser considerados como portadores de derechos y obligaciones e, incluso, reconozcan su capacidad de amar y ser amados por lo que son y no por lo que representan ${ }^{6}$.

En su revisión del concepto de ocio (Elias y Dunnig, 1992), N. Elias plantea expresamente la diferencia entre las actividades de ocio y las relacionadas con el trabajo, a partir de la "referencia» que cada tipo de actividades tiene como guía para la acción ${ }^{7}$. El ocio, dice Elias, pertenece a una esfera en la que se debilitan los procesos de pensamiento relativamente impersonales y no emocionales; al tiempo que se refuerzan los procesos emotivos, y se otorga mayor peso a las funciones de lo que uno hace para si mismo que a las de lo que uno hace por los otros.

Parece evidente suponer que es en esa esfera y en ese espacio de tiempo donde los agentes sociales pueden desarrollar esa "curiosidad ociosa" (idle curiosity) de la que hablaba Veblen; es decir, el tiempo libre es el espacio privilegiado para el desarrollo de un "conocimiento que proviene de la interpretación de los hechos y de la naturaleza del medio ambiente bajo el impulso de una activi-

5 L. Balbo denomina tempo per se "al tiempo que cada uno se concede para la relación consigo mismo, el tiempo de atención de cada uno a sí mismo" (1990: 121).

${ }^{6}$ Sobre este tema, ver N. Elias (1982), La sociedad cortesana, Ed. FCE, México; N. LuHMANN (1984), El amor como pasión, Ed. Península, Barcelona.

7 "En el caso de las actividades laborales todas las decisiones del individuo tienen a otros individuos como marco de referencia, mientras que en las actividades recreativas, el marco de referencia es el propio actor o agente y la búsqueda del placer o la satisfacción personal son algunos de los objetivos básicos». 
dad mental desinteresada» ${ }^{8}$. Pero, sobre todo, la curiosidad ociosa es una clase de conocimiento opuesta al conocimiento de carácter pragmático o utilitario y, en consecuencia, es un concepto equiparable al de excedencia cultural, entendida como el conjunto de «actividades mentales sistemáticamente eximidas de una inmediata significancia práctica, únicamente explorativas y no necesariamente contrarias al orden institucional existente» (Rositi, 1980: 356).

Aun de manera voluntariamente esquemática, puede resumirse que el retroceso del «tiempo de trabajo socialmente necesario" ha supuesto un paralelo incremento de la capacidad (globalmente entendida) de las sociedades modernas para conformar un espacio temporal «libre», dentro del cual se ha configurado un espacio de tiempo en el que los individuos pueden desarrollar su «curiosidad ociosa» o ejercitar el derecho, ahora reconocido, a su «excedencia cultural».

Y de esta cuestión se deriva otra, relativa al uso de este tiempo, que nos conduciría al análisis de las pautas de comportamiento en el tiempo libre, al estudio de la diferenciación social en el consumo del «recurso tiempo», en un detalle que excede con mucho los límites del presente trabajo9. Me limito a señalar alguno de los aspectos más relevantes de la distribución del uso del tiempo libre en las sociedades industriales avanzadas; comenzando por mencionar la tendencia a la privatización de esos mismos comportamientos, tendencia usualmente relacionada con el establecimiento de un creciente desnivel de conocimientos (knowledge-gap), el cual conduciría, a su vez, a una desigualdad paulatinamente más acusada en torno a la capacidad de los agentes sociales para usar y disfrutar de la cultura y el arte.

Pero aludir al alejamiento de los agentes sociales de los espacios públicos, y la consecuente privatización de las prácticas culturales, no presupone condición alguna para dichas prácticas. Acaso, tan sólo, una mayor dependencia de los medios masivos de comunicación, de las necesidades de consumo provocadas por los fabricantes de equipamientos audiovisuales o las industrias culturales. Sabemos qué «dejan» quienes no acuden a espectáculos públicos, quienes no participan de las manifestaciones culturales colectivas, pero resulta muy dificil probar qué obtienen de unos media cada vez más numerosos y capaces de proporcionar una mayor diversidad y pluralidad de mensajes.

Quizás, como recuerda N. Elias, nos encontramos en un periodo en que el tiempo libre ha aumentado más rápidamente que la capacidad de las personas para emplearlo en su ocio. En este sentido, la privatización de la vida en las sociedades industriales avanzadas $-\mathrm{y}$, por ende, la privatización de las pautas de comportamiento en general y las prácticas culturales en particular- puede interpretarse simplemente como una "forma voluntariamente elegida de perder el tiempo».

${ }^{8}$ J. Atkinson Hobson (1978: 11).

9 Sobre estos aspectos puede consultarse I. DomíngueZ, El reparto del tiempo libre, Actas del Congreso de la European Leisure Research Association, Bilbao, España (junio 1992). 
En cualquier caso, no nos hallamos ante un mecanismo que suponga la consideración de los media como un instrumento al servicio de los receptores, en el sentido propuesto por la teoría de los «usos y gratificaciones», que les otorgaba un papel activo y selectivo. Antes bien, tanto el contenido mayoritario de los medios de comunicación masiva como los datos ofrecidos por las encuestas de presupuestos de tiempo o de pautas de comportamiento cultural desmienten cualquier atisbo de "búsqueda activa» por parte de la audiencia de los media. A la rutina de la esfera laboral le corresponde la rutina de un ocio mayoritariamente conformista, basado en el consumo de programas, bienes y servicios producidos masivamente por las diferentes ramas de las industrias culturales. Un tiempo libre en el que, lejos de buscar compensación a la reglamentación de la vida colectiva, se acepta el sometimiento a los ritmos temporales fijados por los media en sus "rejillas de programación", generando relaciones sociales de clara dependencia, obvias aun cuando seamos incapaces de determinar la importancia de una actividad concreta (p. ej., mirar la televisión) a partir de la cantidad de tiempo invertido en ella.

Una posible explicación a esta preferencia que mantiene alejada a la gran mayoría de la población de los fenómenos culturales de carácter público y colectivo, quizá pueda hallarse en la capacidad de los instrumentos de comunicación de masas para ofrecer "normas de comportamiento" ${ }^{10}$, con la celeridad requerida por una sociedad en constante cambio. Estos medios resultan, en este sentido, enormemente más eficaces que los «agentes culturales tradicionales» (teatro, danza, música e, incluso, cine), generalmente dependientes de la financiación pública y, sobre todo, sometidos a la esfera del sistema político.

El resultado de todo ello parece ser un progresivo distanciamiento entre las pautas de comportamiento cultural (de sus prácticas y del nivel de sus equipamientos) de quienes, por estatus social y estilo de vida, son capaces de acceder a las distintas variedades de la oferta cultural y quienes limitan su actividad cultural a la relación con los medios masivos de comunicación. Sólo en el caso de los primeros pueden encontrarse, además, las condiciones para una contingente utilización de ese espacio temporal «libre», dentro del cual es posible desarrollar la «curiosidad ociosa» o la «excedencia cultural» básicas para la creación cultural y artística. O, dicho con otras palabras, nos hallamos ante la implantación de nuevos criterios de desigualdad social.

10 "La televisión reproduce las situaciones cotidianas; representándolas, las transforma en momentos ejemplares, y los dota asi de una canonicidad propia; estos momentos ejemplares, convertidos en cánones, se asumen a su vez como guía de la actuación cotidiana, en un proceso circular al que se denomina "gramaticalización de la cotidianidad"». Casetti; cfr. M. Wolf (1994: 90). 


\section{DESIGUALDAD SOCIAL Y POLÍTICAS CULTURALES}

La desigualdad es, obviamente, una cuestión comparativa. Cuando nos referimos a condiciones de igualdad o desigualdad en cualquier ámbito, estamos estableciendo algún criterio a partir del cual fijar las posiciones relativas que cada actor social ocupa en relación a «algo». Lo que se traduce, si seguimos a $\mathrm{N}$. Bobbio, en la posición que cada individuo ocupa respecto a tres variables que siempre hay que tener en cuenta cuando se introduce el discurso sobre la deseabilidad y viabilidad de la idea de igualdad: $a$ ) los sujetos entre los cuales nos proponemos repartir los bienes o los gravámenes; $b$ ) los bienes o gravámenes que repartir; y c) el criterio por el cual repartirlos (1995).

Pero, además, a esta dificultad intrínseca al concepto de desigualdad se añade la propia condición de lo cultural a la que antes aludíamos y que, como hemos pretendido mostrar en otra ocasión (Domínguez, 1996), está cruzada de significados valorativos cambiantes, que hacen prácticamente imposible la fijación de criterios universalmente aceptados de medida, criterios que permitan su conmensurabilidad. De ahí se deriva un considerable grado de aleatoriedad en la aplicación de las políticas culturales, incapaces de determinar el «umbral de carencia» de los actores sociales en relación a la cultura

Dicho con otras palabras, la desigualdad en materia cultural se expresa - como cualquier otra forma de desigualdad social - mediante atribuciones de sentido socialmente construidas que sirven para ordenar jerárquicamente las diferencias sociales. Es la conversión de las diferencias abstractas en desigualdades concretas, sea en lo cultural o en cualquier otra esfera de lo social, lo que posibilita la generación de estratos, la construcción de un sistema de estratificación social.

En lo cultural, como decíamos más arriba, la idea de una estratificación social tiene una primera expresión en las diferentes capacidades de acceso a los bienes y servicios culturales, así como en el desarrollo diferencial del "gusto», en cualquiera de sus niveles o etapas (gusto, afición, práctica). En ambos casos, la relación entre la noción de estratificación y las pautas de comportamiento cultural suele plantearse de manera unidireccional, siendo éstas - sea cual sea la teoría de la estratificación social que se emplee ${ }^{11}$ - el resultado de la situación o posición social de los individuos, del lugar que ocupan en la estructura social.

Un ejemplo pionero de este modo de análisis lo encontramos en la Teoría de la clase ociosa de T. Veblen, quien en 1899 establecía un vínculo directo entre la posición de clase de los actores sociales (materializada en última instancia en su capacidad pecuniaria) y su capacidad para el consumo y el ocio ostentosos, así como para el acceso, la posesión y el disfrute privilegiados de bienes y servicios culturales. La localización en la estructura de clases determi-

11 Un inteligente repaso a las distintas teorías que se han ofrecido sobre las relaciones entre la cultura y la estratificación social lo encontramos en Crompton (1994). 
na, en la interpretación de Veblen, un uso de los bienes materiales, del espacio y del tiempo sociales que revierte como refuerzo simbólico de la propia posición social.

$\mathrm{Y}$ es preciso añadir que en la actualidad de las sociedades industriales avanzadas, sea por el aumento generalizado del nivel de vida o, si se prefiere, por el alejamiento de buena parte de la población de los umbrales de la subsistencia, las tendencias expresadas por Veblen hace casi un siglo se han reforzado, y ha aumentado la importancia del consumo y del ocio como instrumentos de significación social frente a la producción y el trabajo. Estos procesos, que en lo relativo a las relaciones entre la estructura social y la cultura suponen la emergencia de la noción de "estilos de vida», han dado origen a una segunda tendencia caracterizada por el énfasis puesto en los «elementos» culturales de las clases, desarrollando un "culturalismo» que considera que la cultura es una buena medida autónoma y que las culturas de clase, lejos de constituir reflejos ideológicos de los procesos de clase, los configuran.

Entre ambas interpretaciones figura un buen número de aproximaciones a las relaciones entre cultura y estratificación social basadas en la correspondencia existente entre la "clase ocupacional» y los diferentes estilos de vida o, dicho con otras palabras, las distintas agregaciones formadas en torno al consumo. Más aún, buena parte de las nociones de clase concretan esta posición de clase de los actores sociales en relación con el empleo, con la situación ocupacional de cada individuo ${ }^{12}$.

Pero, dada la situación actual y las tendencias perceptibles en relación al uso social que se da a la cultura en las sociedades posmodernas, esta simple asociación no parece suficiente. Como señala R. Crompton, «el argumento de que el consumo de bienes guarda correspondencia con el nivel social, y el de que la ocupación es un indicador razonable de este nivel social, son generalizaciones que podrían aceptarse. Sin embargo, el interés sociológico por la relación del gusto con los sistemas de estratificación va más allá de la simple demostración de su asociación para explorar los modos en los que el gusto se puede considerar como un recurso que exhiben los grupos dentro del sistema de estratificación con el fin de establecer o ensalzar su situación dentro del orden social» (1994: 211).

Una sociedad que asiste al fin de la «centralidad del trabajo» como instrumento de significación social no puede permanecer impertérrita, sin alterar los valores que sustentan sus metodologías de análisis o sus criterios de asignación de sentido. Y cuando el consumo y el ocio se constituyen en los criterios emergentes de atribución de significados - un fenómeno que, como decimos, no es nuevo, aun cuando ahora, con el fin del trabajo, adquiera toda su relevanciaes preciso aceptar la existencia de una correspondencia entre las formas simbólicas de la acción social y los mecanismos de agregación de los actores sociales,

12 Sobre las recientes formulaciones de las teorías de las clases sociales pueden verse los trabajos de J. Goldthorpe, E. O. Wright y otros, en J. Carabaña (1995) y J. Carabaña y A. de Francisco (1995). 
al margen de sus concretas condiciones de existencia y con independencia de las variaciones de sus respectivos «estilos de vida». No es ésta una propuesta teórica reciente; autores como Bourdieu, Archer o Giddens consideran la "estructura» y la «acción» como parte de una naturaleza «intrínsecamente doble» de la realidad social, en la que lo cultural tiene una notoria incidencia sobre las condiciones de existencia de los actores sociales, influencia paralela a la producida por la posesión de poder o capital económico que puede poseer un individuo o un grupo.

Dicho con otras palabras, es necesario insertar lo cultural en los procesos de diferenciación social, no sólo como resultado de una estratificación social que se concreta en la aparición de mapas de gustos, aficiones o prácticas culturales, sino, incluso, como una variable independiente que influye significativamente en la construcción y consolidación de la posición social (habitus) de clase.

En el primer caso, la estratificación cultural se expresa — como a continuación veremos- en un conjunto de tendencias de uso de la cultura que contribuyen a ahondar las diferencias entre los distintos agregados sociales. En el segundo caso, la estratificación cultural está íntimamente ligada a las dimensiones básicas de la evaluación de una determinada posición social, como el prestigio, la preferencia que dicha posición recibe (el deseo de identificarse con ella, "de ser como» el que la ocupa), y su popularidad, la notoriedad y celebridad asociadas a una posición (Tumin) ${ }^{13}$. En este sentido, como señalábamos al principio de estas líneas, apoyándonos en las palabras de D. Bell, el desarrollo de la sociedad de consumo de masas ha generado tanto una hiperinflación de símbolos como un irrefrenable proceso de mercantilización de los bienes y servicios culturales, inmersos - probablemente de manera definitiva- en un acelerado tratamiento de valorización que los asocia y equipara a otros bienes y servicios de consumo de masas.

Esta inserción de lo cultural en los mecanismos generales de creación de valor, aun cuando obliga a asumir la importancia relativa de lo económico frente a lo cultural en la estructuración y perpetuación de los sistemas de desigualdad social, no significa la admisión inercial de una relación directa (de causa-efecto) entre los niveles de renta y el nivel cultural de los actores sociales; relación que no resulta, en las sociedades occidentales avanzadas, y por motivos de muy diferente índole, tan evidente como pudiera parecer.

En primer lugar, por la difícil consideración del «nivel cultural» de los individuos, no sólo por la compleja mensurabilidad de lo cultural, sino, incluso, por la cambiante determinación del sentido de "lo culto" en sociedades acostumbradas a la variación de la moda; sociedades en las que la «novedad» constituye un valor en sí mismo y en las que, no es conveniente olvidarlo, la relación de los individuos con la cultura reviste la forma de consumo, lo que conlleva el riesgo de que se establezca una falsa equivalencia entre el nivel de

13 Cfr. N. Laurin-Frenette (1976: 193). 
consumo de bienes y servicios culturales y el nivel de cultura de los consumidores.

En segundo lugar, es preciso señalar que la participación del Estado interventor en el ámbito de la cultura ha contribuido a la relajación de las relaciones de causalidad entre las condiciones de vida (y no sólo la variable renta) y el nivel cultural de los individuos. En este sentido, debe consignarse la influencia de las políticas culturales en el incremento de la capacidad de acceso de los individuos a determinadas prácticas, bienes y servicios culturales o, por decirlo con otras palabras, su papel en la «democratización de la cultura». Una cuestión al margen es que los resultados de este esfuerzo político no hayan sido los esperados, que las políticas culturales se desarrollen entre el efectismo de lo espectacular y la ineficacia, y que la desigualdad en materia cultural no haya dejado de aumentar en las últimas décadas y, lo que resulta más grave, mantenga una tendencia creciente.

Los bienes y servicios culturales se han mercantilizado y las prácticas que las generaciones anteriores consideraban «lujosas» — como la ópera o la danza, las vacaciones - son, tanto por efecto de la intervención del Estado como por el relativo alejamiento de los niveles de subsistencia de buena parte de la población, accesibles a casi todos, cuando no se transforman en prácticas «necesarias», en virtud de los atributos de prestigio o calidad que les hayan sido socialmente asignados.

Vista esta cuestión de las relaciones entre la estructura social y la cultura desde una perspectiva tradicional, nos encontraríamos en la obligación de presuponer que el «nivel de cultura» (sea lo que sea que esta expresión signifique) de un individuo o agregado social es el resultado de la influencia de un conjunto de variables o factores sociales - como los niveles de instrucción o de renta, la edad, el sexo, el estado civil, el tamaño del hábitat en el que reside o la relación con la actividad ocupacional - que determinan el uso del tiempo en actividades de carácter cultural y el grado de aprovechamiento que cada actor social obtenga de las mismas.

Sin embargo, el mantenimiento de esta relación de causalidad centrada en la determinación estructural de los comportamientos culturales de los actores sociales supone - a nuestro entender - la asunción acrítica de un conjunto de valores sociológicos pertenecientes al pasado, propios de la sociedad del trabajo $y$, consecuentemente, de difícil aplicación en formaciones sociales posmodernas en las que lo cultural impregna las prácticas de sociabilidad, contribuye a la configuración de «estilos de vida» y, sobre todo, se propugna como instrumento de diferenciación, esto es, como criterio de desigualdad social.

Incluso, no resulta posible establecer una relación de causalidad entre niveles de renta y nivel cultural por la ruptura en la identificación entre un elevado nivel de ingresos y un paralelo nivel de instrucción, considerados ambos como variables sociológicas. Las sociedades más avanzadas son, por paradójico que resulte, las que mantienen un mayor grado de «excedencia cultural» (Rositi), esto es, las que asumen la existencia de un agregado social cada vez más nume- 
roso al que se "permite» pensar con un sentido no inmediatamente práctico. Ello ha provocado el crecimiento global de colectivos más o menos nutridos de estudiantes, técnicos, profesores y artistas, cuyos niveles de instrucción y preparación en algunos casos rozan la excelencia aun cuando sus rentas no sean elevadas.

Pese a todo ello, la idea de una estratificación basada únicamente en criterios de diferenciación cultural, en la que la desigualdad social se ciñera a las diferentes capacidades de acceso o uso de los bienes y servicios culturales, parece absurda ${ }^{14}$. Sobre todo porque lo ocupacional permite aún una relativamente cómoda identificación de las posiciones de clase en relación al poder y, como quería Weber, al mercado. Pero ello no obsta para asumir la importancia de lo cultural como uno de los criterios centrales en los procesos de diferenciación social propios de las sociedades industriales avanzadas, sociedades en las que la difusión de los derechos de ciudadanía (en el sentido expuesto por Marshall ${ }^{15}$ ) y el repetidamente mencionado proceso de pérdida de la centralidad del trabajo han facilitado una alteración de los criterios de diferenciación.

El recurso a criterios de diferenciación de carácter simbólico, común a todas las etapas de la humanidad, tiene, sin embargo, una diferencia evidente con respecto a lo expresado por Veblen o Simmel: la diferenciación cultural no se limita a reproducir la estratificación de clases, sino que contribuye a generarla. Dicho con otras palabras, la estratificación social no es el cauce para la diferenciación de carácter simbólico, en la que las clases o los distintos estratos sociales reproducen sucesivamente los atributos de significado de las clases inmediatamente superiores. Antes bien, como expresa Lipovetski (1987), esos estratos organizados en función de criterios de diferenciación simbólica se constituyen horizontalmente. Las diferencias entre los distintos agregados sociales son, básicamente, diferencias de estilos o modos de vida (Juan, 1991; Scardigli, 1987) en las que que el uso de los bienes simbólicos ocupa una posición dominante, susceptible de ser interpretada como criterio de significación social.

\section{LAS «TIPOLOGÍAS» DE LOS ACTORES CULTURALES}

La importancia que adquiere lo cultural en las sociedades posmodernas es, en consecuencia, fundamental, no sólo como criterio de carácter abstracto, sino, incluso, como mecanismo eficaz para la acción social de los individuos.

${ }_{14}$ R. Crompton subraya que "uno de los argumentos de este libro es que debemos aceptar la idea de Bourdieu de que el capital cultural (que incluye el gusto y el estilo de vida) contribuye de modo importante a la adquisición y el mantenimiento de la posición social de clase. Sin embargo —añade_- la diferenciación cultural nunca puede mantener o explicar por si misma el orden de estratificación" (1994: 250).

15 Sobre este aspecto puede consultarse Domínguez (1994). 
Es el diagnóstico de Allan Bloom (1989), quien percibe en las sociedades posmodernas, y fundamentalmente en la institución universitaria, un proceso de desvinculación con la tradición que se concreta en la pérdida del sistema de valores y su sustitución por patrones valorativos de carácter hedonista y narcisista, incapaces de motivar políticamente a unos individuos dominados por la cultura de la imagen.

En cualquier caso, es urgente señalar que lo ocurrido con la enseñanza universitaria no puede extrapolarse a la esfera de lo cultural. La cultura no resiste la comparación con la enseñanza en su relación con los procesos de estratificación y, al revés que ésta, no entra en el ámbito de la reclamación de igualdad, probablemente por cuanto los atributos culturales no parecen influir en los procesos de selección y movilidad vertical del sistema social, dada la dificultad existente para medir las "capacidades culturales» de los individuos. Por todo ello, al contrario de lo que sucede en torno a la enseñanza, la cultura no resume buena parte de las aspiraciones de diferenciación social que los padres expresan en sus hijos ${ }^{16}$.

El ámbito de la cultura queda, en consecuencia, sometido al flujo del mercado, y se articula en torno a los bienes y servicios producidos por las industrias culturales, en ese espacio sometido a la hegemonía de la cultura de la imagen, y en el que tiene lugar ese notable retroceso de la cultura escrita expuesto por Bloom o por N. Postman. Este último hace extensivo el diagnóstico de Bloom al conjunto de la esfera cultural y escribe un texto (Divertirse hasta morir) que él mismo define como "una investigación y también un lamento sobre el hecho cultural estadounidense más significativo de la segunda mitad del siglo XX: la decadencia de la era de la tipografía y el ascenso de la era de la televisión" (1991: 12).

Postman señala que la mayoría de las ideas modernas sobre la utilización de la inteligencia fueron formadas bajo la influencia de la palabra impresa. La influencia de la imprenta obliga a que el discurso público se caracterice por una disposición coherente y ordenada de hechos e ideas, mientras que a medida que la tipografía se desplaza hacia la periferia de nuestra cultura y la televisión toma su lugar en el centro, la seriedad, la claridad y, sobre todo, el valor del discurso público declinan peligrosamente.

La resolución de esta tendencia en la estructura social nos muestra una progresiva dualización de la sociedad en su relación con la cultura, una pro-

16 Littlejohn (1975) plantea el análisis de la enseñanza, como criterio de estratificación social, a partir de varias características: a) la enseñanza entra en el ámbito de la reclamación de igualdad (entendida como «igualdad de oportunidades»); b) la enseñanza es un instrumento útil para medir la capacidad del sistema social de permitir la movilidad vertical de sus integrantes (su selección); c) asimismo, la enseñanza es el medio idóneo para medir capacidades de los individuos: lingüísticas, conocimientos y habilidades, superación de niveles académicos, etc., abandono y fracaso escolar...; y $d$ ) la enseñanza resume buena parte de las aspiraciones de diferenciación social de los padres. 
pensión al ahondamiento de la brecha ya existente entre los diferentes niveles de aprovechamiento de lo cultural por los diferentes grupos sociales o, si se prefiere, a la constricción de los actores sociales capaces de un pleno disfrute de lo cultural a un reducido grupo de individuos de «estilo de vida cultural».

Un intento de mostrar estas tendencias lo encontramos en el trabajo de O. Donnat (1994) en el que se analiza el conocimiento del mundo del arte y de la cultura como criterio de diferenciación social. Este autor, que ha realizado sucesivos análisis sobre los usos sociales de la cultura entre los franceses, considera que "nunca son los gustos los que mejor caracterizan los medios más cultivados, sino los conocimientos que hacen posible su expresión. Toda práctica cultural exige la acumulación previa de un minimo de informaciones y, en la mayor parte de los casos, de conocimientos" (1994: 15). Es decir, que la condición de "culto" que se concreta en la existencia de unos determinados gustos se deriva de la existencia de un "capital informacional» que, como resulta obvio, depende, a su vez, de la capacidad de acceso a la educación que los distintos individuos hayan disfrutado.

Donnat nos propone un diagnóstico relativo a la esfera de lo cultural, en el que se nos habla de: a) un retroceso absoluto de la cultura consagrada (clásica) entre los más jóvenes; b) un antiintelectualismo que acompaña al déficit de conocimiento de los más jóvenes y que se expresa en el rechazo a los clásicos; y c) el crecimiento de la diversidad del capital informacional de los jóvenes diplomados y su creciente valorización del eclecticismo. Tendencias que muestran la existencia de una ruptura en los mecanismos tradicionales de transmisión de conocimientos. Ruptura no evitada por el alargamiento de la escolaridad y que se concreta en la inexistencia de un corpus canónico de conocimientos básicos. A juicio de Donnat, nos encontramos no sólo con la dificultad de transmitir este corpus a las nuevas generaciones, sino, incluso, con el hecho de que su papel en los mecanismos de distinción es prácticamente nulo y, como consecuencia del cambio habido en los modos de adquisición de los saberes, no cumple ninguna función relevante en la formación de los gustos y en la producción de los modelos de excelencia.

Del análisis efectuado en torno al conocimiento y gusto por personajes célebres, Donnat establece la existencia de varios agregados sociales, en función de su conocimiento de los artistas de la lista: desde los que él mismo denomina excluidos (individuos caracterizados por su carencia de un nivel de instrucción mínimo, de edad anciana, procedentes de hábitat rural, con muy débil capital informacional, relacionados con el mundo a través de la televisión, y con una sociabilidad poco volcada hacia el exterior, fuera de la familia) a los enterados (individuos de edad intermedia, nivel de instrucción alto, con padres de también elevado nivel de instrucción; un agregado cuyos individuos, en su mayoría mujeres, están dotados de un elevado conocimiento de la cultura cultivada) y dispersos (de elevado nivel de instrucción, edad intermedia, es decir, como subraya Donnat, un agregado en el que no se encuentran ni adolescentes ni 
ancianos; en este agregado se hallan los individuos más atentos a la «novedad», los dotados de una mayor diversidad de su capital informacional, quienes realizan el mayor nivel de salidas del hogar y tienen una mayor presencia asociativa; en definitiva; los individuos con unas redes de sociabilidad extrafamiliar más densas) ${ }^{17}$.

Además de este mapa de gustos, aficiones y prácticas culturales, susceptible de ser trasplantado a cualquier sociedad posmoderna sin pérdida de valor descriptivo, y que dibuja una clara tendencia en la construcción de los agregados sociales en relación a la cultura, es preciso añadir otra línea que atraviesa la sociedad en su conjunto, y que se concreta en un intenso proceso de privatización de la experiencia cultural, perceptible en aspectos tales como:

a) El retraimiento al hogar y el alejamiento de los espacios públicos, tendencia que se concreta en la paulatina reducción del número de salidas a espectáculos, recepciones o comidas. Lo que ha convertido a determinados espectáculos como el cine, pero, sobre todo, los epectáculos en vivo (musicales, teatrales o de danza), en acontecimientos excepcionales para la gran mayoría y en el hábito de un reducido grupo de individuos.

b) La preferencia por una experimentación individualizada, facilitada por la difusión de nuevos instrumentos de difusión de los media (walkman, discman) y la aparición de nuevos servicios basados en la red (Internet, servicios de cable), a los que es preciso añadir la «ruptura» de las grandes cadenas de radiotelevisión de carácter generalista.

c) La reducción de las interacciones sociales a las imprescindibles para el mantenimiento de una mínima sociabilidad. Cambio notorio que encuentra su expresión más significativa en el paso de la interacción a la interactividad, en la sustitución de una forma de acción social de los sujetos en sus relaciones con otros sujetos (interacción) por la relación con un sistema mecánico o electrónico (interactividad).

d) El predominio de la «visión de la realidad» transmitida por los massmedia y el alejamiento de la realidad más inmediata, la correspondiente al «mundo de vida» del actor social.

La cultura de las sociedades posmodernas muestra ya —en mayor o menor grado- los rasgos mencionados hasta aquí. Su participación en los procesos de estratificación social no se limita a la existencia de distintas capacidades de acceso a los bienes y servicios culturales, sino que, como hemos intentado exponer en las páginas precedentes, se ha transformado en uno de los vehículos

17 Como es obvio, ésta no es la única tipología existente. Por ejemplo, Crompton señala el trabajo de Savage, quien ha aplicado un mapa de los gustos culturales (o de consumo) a los diferentes sectores de la clase media, construyendo una diferente tipología de consumidores culturales. 
privilegiados para el establecimiento de diferencias jerárquicas entre distintos agregados sociales; diferencias que se resuelven en desigualdades de la capacidad cognitiva y perceptiva de los actores sociales, es decir, sobre su capacidad de acción.

\section{BIBLIOGRAFÍA}

AtKinson Hobson, J. (1978): Veblen, FCE, México, 1. a reimp. (e.o., 1936).

Balbo, L. (1990): Tempi di vita. Studi e proposte per cambiarli, Ed. Feltrinelli, Milano.

BELL, D. (1987): Las contradicciones culturales del capitalismo, Alianza Ed., Madrid, 2. a reimp.

BloOM, A . (1989): El cierre de la mente moderna, Ed. Plaza \& Janés, Barcelona.

Bobbio, N. (1995): Derecha e izquierda, Ed. Taurus, Madrid.

Carabaña, J. (ed.) (1995): Desigualdad y clases sociales. Un seminario en torno a Erik O. Wright, Fundación Argentaria, Madrid.

Carabaña, J., y Francisco, A. de (eds.) (1995): «Teorías contemporáneas de las clases sociales»,

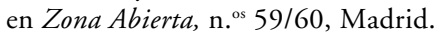

Crane, D. (ed.) (1994): The sociology of culture. Emerging theoretical perspectives, Blackwell, Oxford.

Crary, J. (1994): «Los mitos del ciberespacio», en El Viejo Topo, n. o 74, Barcelona.

Crompton, R. (1994): Clase y estratificación, Ed. Tecnos, Madrid.

DomíngueZ, I. (1994): "Ciudadanía, participación y cultura en sociedades avanzadas», en Inguruak, n. ${ }^{\circ}$ 9, Bilbao.

- (1996): «Las medidas de la cultura», en Actas del III Congreso Vasco de Sociología, Asociación Vasca de Sociología, Bilbao.

Donnat, O. (1994): Les français face a la culture, La Documentation Française, París.

Elias, N., y Dunning, E. (1992): Deporte y ocio en el proceso de la civilización, FCE, Madrid.

Giddens, A. (1993): Consecuencias de la modernidad, Alianza Ed., Madrid.

- (1995): La constitución de la sociedad, Amorrortu Ed., Buenos Aires.

LAURIN-FrenetTe, N. (1976): Las teorías funcionalistas de las clases sociales, Ed. Siglo XXI, Madrid.

LiTtLejHON, J. (1975): La estratificación social, Alianza Ed., Madrid.

LYON, D. (1995): El ojo electrónico, Alianza Ed., Madrid.

MelischeK, G.; Rosengren, K. E., y STAPpers, J. (1984): Cultural indicators: An international symposium, Verlag der Österreichischen Akademie der Wissenschaften, Viena.

Postman, N. (1991): Divertirse hasta morir. El discurso público en la era del "show busines», Ed. de la Tempestad, Barcelona.

Rositi, F. (1980): Historia y critica de la cultura de masas, Ed. G. Gili, Barcelona.

Veblen, T. (1987): Teoría de la clase ociosa, Ed. Orbis, Barcelona.

Willians, R. (1982): Cultura. Sociología de la Comunicación y del Arte, Ed. Paidós, Barcelona.

Wolf, M. (1994): Los efectos sociales de los media, Ed. Paidós, Barcelona. 


\begin{abstract}
The objective of this work is multiple: $a$ ) By a part, to propose a reflection on the space that in the daile life of the advanced industrial societies is reserved for the relationship of the individuals with the culture and the art. b) As objective second, we intend to formulate some considerations on the public use and/or private of that relationship of the individuals with the culture and the art. For this, we propose ourselves to show some charateristic of the evolution of that "temporary space» that we designate "free time», as well as of the varieties and conditions of the use that is granted to him/her/you in some societies in those which all the indicia indicate to us the end of a cycle, characterized it in wheel to the work, understood so much as development instrument, as mechanism of differentiation and social meaning. c) And, finally, we propose an analysis of the insert of what is cultural in the processes of differentiation social, not only in the wake of a social stratification that is concretized in the appearance of pleasure maps, interest or cultural practices but, even, as an independent variable that influences significantly the construction and consolidation of the social position of class.
\end{abstract}

